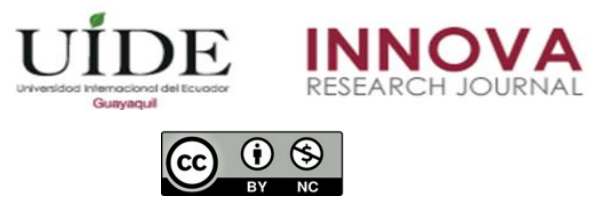

INNOVA Research Journal, ISSN 2477-9024

(Septiembre-Diciembre 2020). Vol. 5, No.3 pp. 209-232

DOI: https://doi.org/10.33890/innova.v5.n3.2020.1436

URL: http://revistas.uide.edu.ec/index.php/innova/index

Correo: innova@uide.edu.ec

\title{
La ley de Okun en Ecuador. Un Análisis de Cointegración, 2007-2019
}

\section{Okun's law in Ecuador. A Cointegration Analysis, 2007-2019}

Diego Ontaneda Jiménez

https://orcid.org/0000-0003-2601-2782

Universidad de Cuenca, Ecuador

Autor por correspondencia: diego.ontanedaj@ucuenca.edu.ec

Fecha de recepción: 25 de mayo del 2020 - Fecha de aceptación: 11 de agosto del 2020

\section{Resumen}

El estudio analiza la ley de Okun para Ecuador durante 2007.I-2019.IV con dos metodologías de cointegración, el método de Johansen \& Juselius (1990) y el enfoque Autorregresivo de Rezago Distribuido (ARDL) propuesto por Pesaran \& Shin (1995) y Pesaran, Shin \& Smith (2001) . Los resultados empíricos indican que en el largo plazo el desempleo tiene un efecto negativo, estadísticamente significativo sobre la producción. El análisis de cointegración señala que un punto porcentual adicional en la tasa de desempleo disminuye la producción en $2.06 \%$ en el caso del enfoque de Johansen \& Juselius (1990) y de $2.09 \%$ con el enfoque ARDL. Los resultados indican una relación bidireccional entre la producción y el desempleo, y señalan que, frente a fluctuaciones en el sistema, el efecto en el desempleo registra su nivel máximo en el quinto trimestre, mientras que el producto alcanza su punto máximo en el tercer trimestre. Esto sugiere que los hacedores de política deben considerar el impulso de la producción como una estrategia clave para combatir el desempleo y el elevado costo de la desocupación en términos de producto, y que los efectos de las políticas que buscan promover la economía van a transmitirse a lo largo del tiempo.

Palabras clave: ley de Okun; Ecuador; cointegración; producción; desempleo

\section{Abstract}

The study analyzes Okun's law for Ecuador during 2007.I-2019.IV with two cointegration methodologies, the Johansen \& Juselius (1990) method and the Autoregressive Distributed Lag (ARDL) approach proposed by Pesaran \& Shin (1995) and Pesaran, Shin \& Smith (2001). The empirical results indicate that in the long term unemployment has a statistically significant negative effect on production. The cointegration analysis indicates that an additional percentage point in the unemployment rate decreases production by 2,06\% in the case of the Johansen \& Juselius (1990) approach and by 2,09\% with the ARDL approach. The results indicate a bidirectional relationship between production and unemployment, and point out that when faced with fluctuations in the system, the effect on unemployment registers its maximum level in the fifth quarter, while the product reaches its maximum point in the third quarter. This suggests that policy makers should consider boosting production as a key strategy to combat unemployment and the high cost of unemployment in terms of output, and that the effects of policies that seek to promote the economy will be transmitted to the long of the time. 
Key words: Okun's law; Ecuador; cointegration; production; unemployment

\section{Introducción}

Entre los años 2007 y 2019, Ecuador presentó marcadas fluctuaciones de la tasa de desempleo, que osciló entre $8.83 \%$ en el primer trimestre del 2007, un $9.10 \%$ en el primer trimestre del 2010 y un $4.54 \%$ en diciembre del 2014, en el que registró su nivel más bajo. Posteriormente, el desempleo incrementó a $7.35 \%$ en el primer trimestre del 2016, y a partir de entonces ha presentado una tendencia decreciente, aunque en el 2019 se observa un claro incremento. Estas variaciones en el desempleo claramente se han visto reflejadas en cambios en el nivel de producción. El incremento del producto se ha correspondido con la disminución del desempleo y viceversa. La relación entre los cambios en el producto y el desempleo es una regularidad empírica que se conoce como la ley de Okun.

No obstante, durante los últimos años en Ecuador se han tenido lugar marcadas fluctuaciones económicas, conjuntamente con importantes cambios en la política y legislación en el mercado laboral. A excepción del año 2009, se registra una fuerte expansión económica hasta 2014, un episodio de contracción relacionado con condiciones externas adversas hasta el segundo trimestre del 2016, y una recuperación hasta el final del período analizado ${ }^{1}$ (Gráfico 1). Al mismo tiempo, se observan cambios institucionales en el mercado laboral, como el incremento sostenido del valor real del salario mínimo (Wong, 2019) y la introducción de una serie de reformas laborales que buscaban reducir la flexibilidad laboral, como la eliminación de la intermediación laboral (OIT, 2014).

La idea básica de la ley de Okun es que una reducción en el desempleo tiene un costo en términos de producción. Okun (1963) analizó empíricamente la relación entre la tasa de desempleo y la producción para la economía de Estados Unidos. El autor relacionó los cambios en la demanda agregada a las fluctuaciones de la producción alrededor de su producto potencial. Los cambios en la demanda agregada generan movimientos en el producto que provocan que las empresas contraten o despidan trabajadores. Esto, a su vez, causa variaciones en la demanda de trabajo y, consecuentemente, cambios en la tasa de desempleo con respecto a su tendencia de largo plazo. Concretamente, la ley postula que un incremento de $1 \%$ en la tasa de desempleo conduce a una reducción de alrededor de $3 \%$ en el crecimiento del producto real.

Con respecto a la literatura de Ecuador, lo que se conoce de la relación dinámica entre producto y desempleo en el contexto actual es más bien escaso, y no hay un consenso con respecto a la magnitud del coeficiente de Okun. Por ejemplo, Briceño, Dávila, \& Rojas (2017) estiman la ley de Okun para Ecuador a partir de una regresión simple con observaciones anuales de 1991-2014 y no encuentran evidencia a favor de esta teoría. Desde el punto de vista metodológico, la principal limitación del estudio es el número de observaciones que considera y el análisis no tiene en cuenta la evidencia de que las series de producto y desempleo pueden presentar procesos de raíz unitaria, lo que genera un problema de regresión espúrea. Adicionalmente, no se tiene en cuenta la dinámica en la relación entre el producto y el desempleo, en donde su comportamiento puede ser el resultado de sus valores actuales y pasados. Rodríguez, Hurtado, \& Vega (2017) analizan la ley de Okun para Ecuador con datos

${ }^{1}$ El BCE (2020b) identifica tres ciclos económicos del Producto Interno Bruto entre 2007 y 2019. 
trimestrales durante el periodo 2006.I-2015.IV a través de un modelo de Vector Autorregresivo (VAR). Este estudio presenta la limitación de que considera información de empleo para el año 2006, la cual no es comparable con las series de empleo obtenidas a partir del 2007. Los autores no analizan la ley de Okun a partir de la relación entre los componentes cíclicos del desempleo y producto, y no determinan una relación de equilibrio de largo plazo, lo que implica que los resultados del modelo VAR pueden estar sujetos a un problema de regresión espúrea. Adicionalmente, la revisión de estudios a nivel internacional permite concluir que la relación entre el producto y desempleo está condicionada por varios factores y no cabría esperar que sea la homogénea entre los diferentes periodos y países.

En este sentido, el presente estudio amplia el análisis de diferentes formas. Primero, abarca un periodo de estudio caracterizado por cambios económicos significativos, escasamente analizado por estudios previos. Segundo, analiza la relación dinámica de la producción y el desempleo a partir de funciones de impulso respuesta y estudia la dirección de causalidad a través de pruebas de causalidad de Granger. Tercero, se determina la relación de equilibrio entre la producción y el desempleo a partir de sus fluctuaciones cíclicas y considera el método de Johansen \& Juselius (1990) basado en un modelo de Vector de Corrección de Error (VEC) que tiene en cuenta la simultaneidad en la relación de las variables, y que sus efectos se transmiten a lo largo de un determinado número de períodos. Cuarto, se aplica el enfoque Autorregresivo de Rezago Distribuido propuesto por Pesaran \& Shin (1995) y Pesaran, Shin, \& Smith (2001) que tiene ventajas frente a las metodologías aplicadas en estudios previos, particularmente en muestras pequeñas, y no ha sido considerado para analizar la relación entre el producto y desempleo en Ecuador. Para determinar la tendencia de largo plazo de las series se utiliza el filtro de Hodrick \& Prescott (1997) y se analiza la robustez de los resultados a través del filtro band-pass propuesto por Baxter \& King (1995). Así, el periodo de estudio considerado y la aplicación de diferentes metodologías de cointegración hace posible obtener un soporte consistente para las hipótesis analizadas y robustecer los resultados.

El alcance del estudio es bastante específico, ofrece una estimación puntual de la relación de largo plazo entre el desempleo y producto para Ecuador, y analiza la relación dinámica entre estas variables a partir de un análisis de impulso respuesta y pruebas de causalidad de Granger. El análisis de estos factores tiene importantes implicaciones de política. Estos resultados pueden servir de criterio para los hacedores de política para evaluar el costo de las elevadas tasas de desempleo en términos del producto. Señala la capacidad de respuesta del mercado laboral ecuatoriano a cambios de la producción, y permite analizar cómo las políticas destinadas a promover la economía y el empleo pueden repercutir a lo largo del tiempo.

El artículo se organiza de la siguiente forma. En la segunda sección se presenta una breve descripción de los antecedentes de la economía ecuatoriana. La tercera sección presenta una revisión de estudios empíricos que analizan la relación entre producción y desempleo. La cuarta sección presenta el marco teórico de referencia. La sección cinco desarrolla el análisis empírico y finalmente, en la sección seis, se señalan algunas observaciones finales y se discute implicaciones de política asociadas a la relación desempleo-producto. 


\section{Breve descripción de antecedentes}

Al igual que otros países de la región, a mediados de la década de 1990, el país experimentó un intenso proceso de reformas estructurales. Ecuador había liberalizado los tipos de cambio y de interés, el comercio y los movimientos de capitales, eliminado subsidios, desregulado parcialmente el sistema financiero y flexibilizado el mercado laboral (Larrea, 2004). Entre los años 1998-1999, Ecuador experimenta la crisis económica y financiera más profunda de los últimos años. La crisis estuvo asociada principalmente a choques externos como la caída del precio del petróleo, principal producto de exportación, a consecuencias climáticas relacionadas con el fenómeno de El Niño y al quiebre de la mayoría de bancos privados del país (Larrea, 2004). Durante el segundo quinquenio de los años 90, la economía ecuatoriana creció a una tasa del 0.92\%. En 1999, el Producto Interno Bruto (PIB) decreció en términos reales en aproximadamente -4.86\% (BCE, 2019). En medio de la crisis, el gobierno decretó la dolarización de la economía en el año 2000.

La crisis se manifestó en un marcado incremento de la pobreza, el desempleo, subempleo y una masiva migración internacional. Entre 1998 y 1999 la tasa de pobreza urbana pasó del $54.52 \%$ a 58.49\% (SIISE, 2019), la tasa de desempleo incrementó en 3 puntos porcentuales (11.5\%-14.4\%) y la tasa de subempleo se ubicó en alrededor del 68\% (BCE, 2019b). En el año 1999, la migración internacional en Ecuador creció en 114\%, y se estima que entre 1996 y 2010 aproximadamente 610,572 ecuatorianos migraron al exterior (Mendieta Muñoz, 2015). Posterior a la crisis, y en el marco del nuevo esquema monetario, la situación económica comenzó a estabilizarse progresivamente. Con la excepción de 2009, 2015 y 2016, el crecimiento del PIB en Ecuador ha sido muy consistente. Entre 2003 y 2014 la tasa de crecimiento promedio anual del PIB fue de $4.67 \%$ y la tasa de desempleo ha registrado un progresivo decrecimiento de 5.51 puntos, pasando de 9.3\% a 3.8\% de la Población Económicamente Activa (PEA). Los años 2009, 2015 y 2016 fueron periodos de crecimiento lento o negativo asociados con condiciones externas adversas, como la marcada reducción del precio del petróleo.

\section{Revisión de estudios empíricos con respecto a la ley de Okun}

Un amplio número de estudios empíricos ha analizado la ley de Okun para diferentes economías. Se observa que la relación entre el producto y desempleo ha sido estudiada a partir de diferentes especificaciones y métodos de estimación, incluyendo series temporales nacionales y regionales, y datos de panel de diferentes países.

Los estudios pioneros analizaron empíricamente la ley de Okun principalmente para el caso de Estados Unidos. Se ha llegado a la conclusión de que en este país el coeficiente de la ley de Okun sería un poco más de 2\% (Attfield \& Silverstone, 1997; Nourzad \& Almaghrbi, 1996; Prachowny, 1993). Posteriormente, el análisis se amplió para determinar si la ley de Okun se puede aplicar a otros países con diferentes estructuras económicas. Por ejemplo, el estudio de Ball, Furceri, Leigh, \& Loungani (2019) al analizar la ley de Okun para un conjunto de 71 países determina que el coeficiente de Okun es aproximadamente la mitad del tamaño en países en desarrollo que en países desarrollados. Un ejemplo de estudios enfocados en economías específicas es Chavarín (2001) que analiza el caso de México para el periodo 1987.I-2000.I y determina que el coeficiente de la ley de Okun es de alrededor de $2.4 \%$. Islas \& Cortez (2013) 
que al utilizar un modelo bivariado para determinar los componentes permanentes y transitorios del producto y desempleo durante 1987.I-2008.IV en México, encuentran un coeficiente mucho menor, una variación del desempleo de 1 punto porcentual genera una variación en la producción, en promedio, de -1,66 puntos porcentuales. Adanu (2005) al analizar la ley de Okun para las provincias canadienses durante el periodo 1981-2001 a partir de las fluctuaciones cíclicas del producto y el desempleo determina que el coeficiente de Okun es de -1.58 y-1.32 cuando se aplica el filtro de Hodrick-Prescott y de tendencia cuadrática para eliminar la tendencia de las series. En un estudio similar, Apergis, Rezitis, \& Rezitis (2003) estiman el coeficiente de Okun para varias regiones de Grecia durante el periodo 1960-1997, utilizando dos métodos para calcular el componente cíclico de las series. Los resultados indican que el coeficiente de Okun fluctúa entre 1.03 y 2.79 para el filtro de Hodrick-Prescott, y 1.14 y 2.81 para el filtro de Baxter-King.

Los estudios que buscan determinar una relación de equilibrio de largo plazo entre la producción y desempleo señalan que el coeficiente de Okun varía según el horizonte temporal analizado (corto o largo plazo). En esta línea, Zagler (2003) utiliza un Modelo de Vector de Corrección de Error (VECM) para analizar la relación entre el crecimiento económico y desempleo para cuatro países europeos, Francia, Alemania, Italia y Reino Unido. Los autores determinan que en el largo plazo el crecimiento económico y el desempleo están positivamente correlacionados, lo que no concuerda con la ley de Okun. Aunque el autor determina que en el corto plazo un incremento en el desempleo conduce a una reducción del crecimiento económico. Flórez, Pulido-Mahecha, Ramos, \& Ramos-Veloza (2018) utilizan un modelo de VECM para determinar la relación de largo plazo entre la tasa de desempleo y el producto en Colombia entre 1984-2016. Los resultados confirman la ley de Okun para el caso colombiano. Al analizar las no linealidades en la relación de cointegración, los resultados sugieren un régimen alto, en el cual el incremento de $1 \%$ en el producto reduce la tasa de desempleo en promedio en 0.6 puntos porcentuales y un régimen bajo en el cual la respuesta es en promedio de 0.2 puntos porcentuales. Abu (2017) utiliza un modelo ARDL para estudiar la ley de Okun en Nigeria entre 1970-2014 considerando adicionalmente el papel de los precios del petróleo. Los resultados indican que en el largo plazo el desempleo tiene un efecto negativo y significativo sobre el crecimiento económico.

Una pregunta más reciente analizada por los estudios empíricos es cómo diferentes factores pueden mediar en la relación entre el desempleo y la producción. Por ejemplo, el estudio de Islas \& Cortez (2019) explica que la flexibilidad laboral puede afectar el grado de respuesta del desempleo ante cambios en la producción de diferentes formas. La mayor flexibilidad laboral se traduce en mayores fluctuaciones en el empleo si implica que las empresas puedan contratar y despedir a sus trabajadores en línea con sus operaciones. Si la flexibilidad laboral determina esquemas de contratación más flexibles de empleo, como es el caso de la informalidad laboral, entonces las variaciones de la producción afectarían el desempleo en menor medida. Asimismo, Loria Diaz De Guzman, Ramírez Guerra, \& Salas (2015) analizan como las políticas de flexibilización laboral pueden incidir sobre el nivel de desempleo a partir de un modelo basado en la ley de Okun en México entre 1997.III y 2014.I. Los autores utilizan un modelo de VECM y determinan una relación negativa entre la producción y desempleo, y que la flexibilidad laboral aumenta el desempleo. 
La revisión de estudios a nivel internacional permite inferir que, si bien la literatura generalmente respalda la validez de la ley de Okun, los resultados señalan que existe una marcada heterogeneidad en la magnitud del coeficiente de Okun entre los diferentes países.

\section{Marco teórico de referencia}

Okun (1963) analizó la relación entre el desempleo y producto a partir de tres métodos. Primero, los cambios en la tasa de desempleo se relacionan con cambios en el producto real. Segundo, las variaciones en la tasa de desempleo con respecto a su nivel de equilibrio dependen de las desviaciones del producto de su nivel potencial. Tercero, la tasa de desempleo se relaciona a una tendencia de tiempo y al producto real observado. En todos los casos la variable dependiente es la tasa de desempleo y la variable explicativa una medida de producción.

Siguiendo a Barreto \& Howland (1993) y Chavarín (2001) se propone la estimación de una regresión inversa, en la cual, la variable dependiente es el producto y la variable explicativa la tasa de desempleo. Se considera una especificación utilizada frecuentemente en la literatura (Adanu, 2005; Apergis \& Rezitis, 2003; Chavarín, 2001), en la que la producción cíclica se relaciona con el desempleo cíclico de la siguiente forma:

$$
y_{t}-y_{t}^{*}=\alpha+\beta\left(u_{t}-u_{t}^{*}\right)
$$

Donde el subíndice $t$ se refiere al periodo de tiempo, $y_{t}-y_{t}^{*}$ es la producción cíclica expresada en logaritmos, y $u_{t}-u_{t}^{*}$ es el desempleo cíclico. De acuerdo con este modelo, los cambios en el producto real con respecto a su nivel potencial están relacionados con las fluctuaciones cíclicas del desempleo. El coeficiente de la variable desempleo mide el costo en términos de producto, del incremento de la tasa de desempleo. Si la ley de Okun es válida, el coeficiente $\beta$ es negativo, así por cada punto porcentual de incremento de la tasa de desempleo, el producto se reducirá en $\beta$ porciento.

\section{Análisis empírico}

Esta sección abarca los resultados empíricos. En la primera subsección se describe la fuente de los datos y se presentan un análisis preliminar de las variables. La segunda subsección realiza las pruebas de raíz unitaria de las series, y la estimación empírica de la ley de Okun se presenta en la tercera subsección.

\section{Datos}

El análisis empírico utiliza datos trimestrales sobre producción medida por el PIB real con precios del año 2007, y la tasa de desempleo abierto expresada como porcentaje de la Población Económicamente Activa para el periodo 2007.I-2019.IV, lo que responde a la disponibilidad y comparabilidad de la información. Los datos provienen del Banco Central del Ecuador. Las series fueron desestacionalizadas a través de la metodología Tramo/Seats. Se utiliza el filtro de Hodrick-Prescott (HP) para descomponer las series en sus componentes de tendencia y cíclico. 
La figura 1 presenta la evolución del PIB y la tasa de desempleo durante el periodo de estudio. Con respecto al PIB se identifican dos periodos clave. El periodo 2007-2014, se caracteriza por un rápido crecimiento, a excepción del año 2009 debido a la crisis internacional. La expansión económica se asocia a una mayor participación del estado en la economía, a través de la expansión del gasto gubernamental en diferentes áreas como infraestructura que entre estos años incrementó con respecto al PIB en 3.37 puntos porcentuales (10.93-14.30) (BCE, 2020a) y a la mejora de los términos de intercambio debido al auge de los precios del petróleo. El periodo 2014-2019, se caracteriza por una marcada contracción de la economía que se asocia a la caída del precio del petróleo desde el 2014. Estos factores han significado que en 2015 la economía creciera en $0.10 \%$ y en 2016 decreciera en $-1.23 \%$, la tasa de crecimiento más baja desde la crisis de finales de la década de 1990. Un comportamiento similar se observa en la evolución de la tasa de desempleo. Se distingue una clara reducción del desempleo hasta el año 2014, a partir de entonces, la tasa de desempleo incrementó hasta alcanzar su nivel máximo en 2016.I. Después de este periodo, se registra un progresivo decrecimiento del nivel de desocupación, aunque en los últimos años se observa una evidente reversión de la tendencia inicial.

La figura 2 presenta el componente cíclico del PIB y la tasa de desempleo obtenido a partir del filtro de HP. El gráfico muestra claramente una relación inversa entre la producción y el desempleo, no obstante, la dirección de la relación no se puede inferir directamente.

\footnotetext{
${ }^{2}$ Cabe señalar que en el año 2016 el país sufrió los efectos de un terremoto de 7,8 grados en la escala de Richter en la costa norte y de acuerdo con Senplades (2016) su impacto en la tasa de crecimiento del PIB fue de $-0,7$ puntos porcentuales.
} 


\section{Figura 1}

Detalla la evolución del Logaritmo del PIB y la tasa de desempleo.

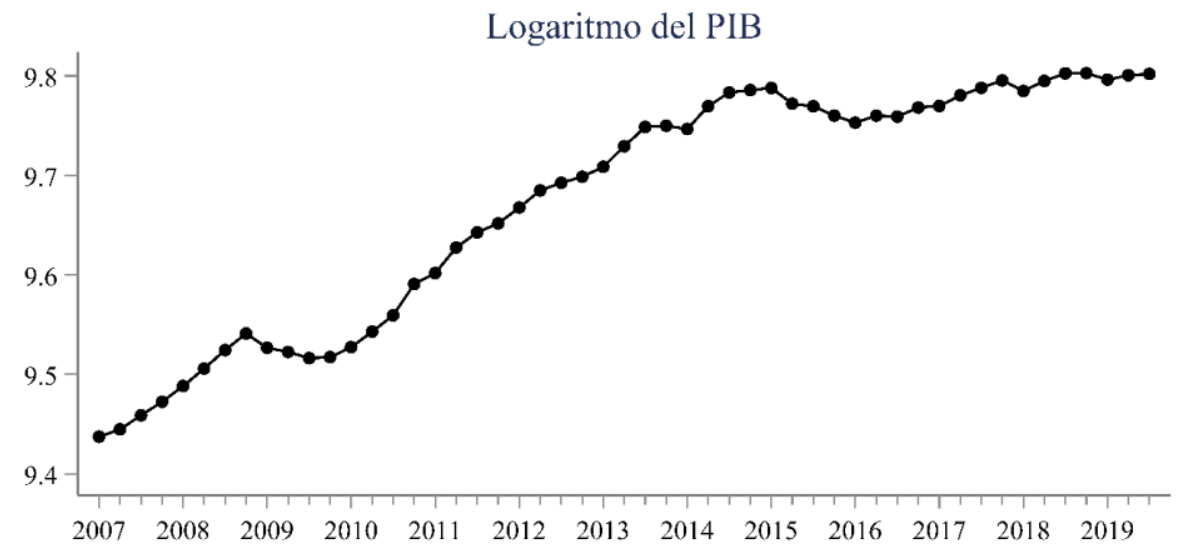

Tasa de desempleo

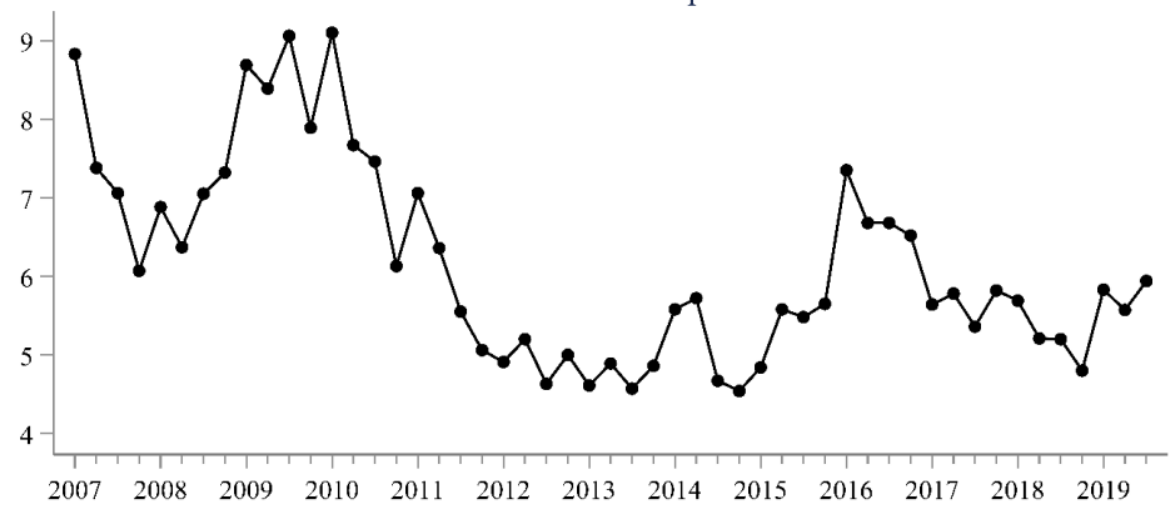

Fuente: Elaboración propia con base en Banco Central del Ecuador. 
Figura 2

Detalla el componente cíclico del PIB y la tasa de desempleo.

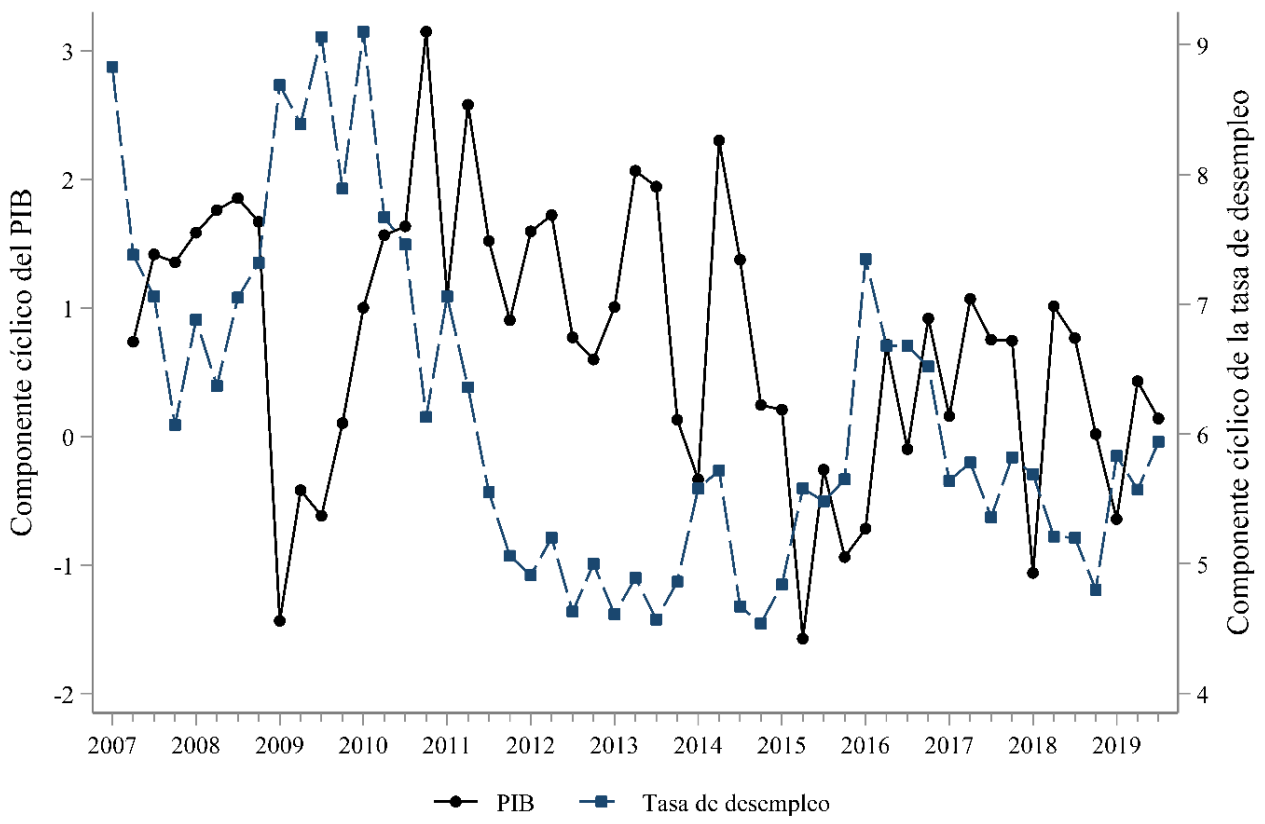

Se utiliza un parámetro de suavizamiento de 1600.

Fuente: Elaboración propia con base en Banco Central del Ecuador.

\section{Pruebas de raíz unitaria}

Para llevar a cabo la prueba de raíz unitaria, se utilizan tres métodos incluyendo la prueba de Dickey-Fuller aumentada (ADF), Phillips-Perron (PP) y Dickey-Fuller con mínimos cuadrados generalizados propuesta por Elliott, Rothenberg y Stock (ERS). La prueba ERS es una prueba de raíz unitaria más eficiente frente a procesos estacionarios altamente persistentes en el tiempo, en comparación con las pruebas de raíz unitaria ADF y PP (Elliott, Rothenberg, \& Stock, 1992). Las pruebas ADF, PP y ERS prueban la hipótesis nula de que una serie es una raíz unitaria frente a la hipótesis alternativa de que la serie es un proceso estacionario. En todas las pruebas se incluyó una constante, pero no tendencia. Al nivel de significancia habitual del 5\% y $1 \%$ no se rechaza la hipótesis de que las series del PIB y la tasa de desempleo son no estacionarios en sus niveles. La evidencia muestra que todas las variables en niveles son integradas de orden uno I(1), mientras que las primeras diferencias son series $\mathrm{I}(0)^{3}$ (Tabla 1).

\footnotetext{
${ }^{3}$ En el anexo 1 se presentan las pruebas de raíz unitaria aplicadas a las series originales. Se llega a la conclusión de que las variables en niveles son integradas de orden uno I(1), y sus primeras diferencias son series I(0).
} 
Tabla 1.

Resultados de las pruebas de raíces unitarias (incluye intercepto)

\begin{tabular}{|c|c|c|c|c|c|c|}
\hline & ADF & & PP & & ERS & \\
\hline Variable & Nivel & $\begin{array}{l}\text { Primera } \\
\text { diferencia }\end{array}$ & Nivel & $\begin{array}{l}\text { Primera } \\
\text { diferencia }\end{array}$ & Nivel & $\begin{array}{l}\text { Primera } \\
\text { diferencia }\end{array}$ \\
\hline Producción & $-2.620(1)$ & $-4.237(0) * * *$ & $-2.326(3)$ & $-4.277(3) * * *$ & $-2.287(1)^{*}$ & $-4.281(0) * * *$ \\
\hline Tasa de desempleo & $-2.24(1)$ & $-10.047(0) * * *$ & $-3.480 *(3)$ & $-9.880(3) * * *$ & $-1.933(1)^{*}$ & $-5.513(0) * *$ \\
\hline
\end{tabular}

En vista de que el periodo de estudio abarca la crisis financiera internacional de 2009 y el descenso del crecimiento económico registrado en los años 2014 y 2015, se utiliza la prueba de raíz unitaria de Zivot \& Andrews (2002) con cambio estructural endógeno. Si existe una ruptura en la tendencia determinística, entonces las pruebas de raíz unitaria conducen a una conclusión errónea de que la serie presenta un proceso de raíz unitaria cuando de hecho es estacionaria (Maddala \& Kim, 1998)

Los resultados sugieren que no se puede rechazar la hipótesis nula según la cual las series del PIB y desempleo presentan un proceso de raíz unitaria y, por lo tanto, sus niveles no son estacionarios (Tabla 2). Estos resultados confirman lo encontrado por las pruebas de raíz unitaria sin cambio estructural para las dos series. Cabe mencionar que la prueba de cambio estructural endógeno indica la presencia de un cambio en la serie de producción en 2011.II en el intercepto, y en 2014.III en la tendencia. En el caso de la tasa de desempleo, el cambio se presentaría en 2011.III y 2009.I en el intercepto y la tendencia, respectivamente. Estos resultados son coherentes con la evolución de las variables observada en los Gráficos 1 y 2, y concuerdan con episodios importantes de la economía ecuatoriana, la crisis financiera internacional y desaceleración del crecimiento registrada durante los últimos años.

Tabla 2.

Resultados de las pruebas de raíces unitarias de Zivot y Andrews one-break

\begin{tabular}{llll}
\hline Variable & Ruptura & t $(\mathbf{k})$ & Periodo de ruptura \\
\hline Producción & Intercepto & $-3.333(1)$ & 2011. II \\
\hline Tasa de desempleo & Tendencia & $-2.757(1)$ & 2014. III \\
\hline & Intercepto & $-3.931(2)$ & 2011. III \\
\hline
\end{tabular}

$* \mathrm{p}<0.10, * * \mathrm{p}<0.05, * * * \mathrm{p}<0.01$. Los valores críticos para la prueba de Zivot y Andrews considerando cambio en el intercepto son $-5.34,-4.80$ y -4.58 y considerando cambio en la tendencia $-4.93,-4.42$ y -4.11 para los niveles de significancia de 1\%,5\% y $10 \%$ respectivamente. Entre paréntesis se presenta la estructura de rezago óptima de acuerdo al AIC.

Fuente: Elaboración propia. 


\section{Contrastación empírica de la ley de Okun}

\section{Modelo estático}

La tabla 3 presenta la estimación Mínimos Cuadrados Ordinarios (MCO) para el modelo estático de la ecuación (1). Los resultados corresponden a las estimaciones del modelo utilizando dos tipos de filtros para descomponer las series en su componente cíclico y tendencia, el filtro de Hodrick-Prescott y Baxter-King.

Los resultados indican que el incremento de un punto porcentual (p.p) en la tasa de desempleo trimestral, provoca una disminución del PIB, en promedio en $1.36 \%$ cuando se utiliza el filtro HP. Cuando se emplea el filtro de Baxter-King, la respuesta del producto al incremento del desempleo incrementa a $2.19 \%$,

Tabla 3.

Coeficientes de Okun estimados

\begin{tabular}{lll}
\hline & Hodrick-Prescott & Baxter-King \\
\hline Desempleo & $-0.014 * * *$ & $-0.022^{* * *}$ \\
\hline & $(0.002)$ & $(0.003)$ \\
\hline Constante & $-1.230 \mathrm{e}^{\wedge}-11$ & 0.004 \\
\hline R cuadrado & $(0.002)$ & $(0.002)$ \\
\hline F (Prob $>$ F) & 43.42 & 0.66 \\
\hline $\mathbf{N}$ & 52 & $52.89(0.00)$ \\
\hline
\end{tabular}

$* \mathrm{p}<0.10, * * \mathrm{p}<0.05, * * * \mathrm{p}<0.01$. Entre paréntesis se presentan los errores estándar.

Fuente: Elaboración propia.

\section{Modelo dinámico: Modelo de Vector Autorregresivo y modelo de Vector de Corrección de Error (MVEC)}

El análisis parte de la especificación de un modelo de Vector Autorregresivo (VAR) de la siguiente manera:

$$
X_{t}=\alpha+\Pi_{1} X_{t-1}+\Pi_{2} X_{t-2}+\cdots+\Pi_{p} X_{t-p}+\varepsilon_{t}, t=1, \ldots, T
$$

Donde $X$ es un vector de $\mathrm{N}$ variables, $t$ el periodo de tiempo y $p$ es la longitud del rezago. Por ejemplo, para las variables $y_{t}$ y $u_{t}$ con dos rezagos, el modelo VAR(2) tiene la forma:

$$
\begin{aligned}
\left(\begin{array}{l}
y_{t} \\
u_{t}
\end{array}\right)=\left(\begin{array}{l}
\alpha_{11} \\
\alpha_{21}
\end{array}\right) & +\left(\begin{array}{ll}
\pi_{11} & \pi_{12} \\
\pi_{21} & \pi_{22}
\end{array}\right)\left(\begin{array}{l}
y_{t-1} \\
y_{t-2}
\end{array}\right)+\left(\begin{array}{ll}
\gamma_{11} & \gamma_{12} \\
\gamma_{21} & \gamma_{22}
\end{array}\right)\left(\begin{array}{l}
u_{t-1} \\
u_{t-2}
\end{array}\right) \\
& +\left(\begin{array}{l}
\varepsilon_{1 t} \\
\varepsilon_{2 t}
\end{array}\right)
\end{aligned}
$$

Donde $\operatorname{cov}\left(\varepsilon_{1 t}, \varepsilon_{2 s}\right)=\sigma_{12}$ para $t=s ; 0$ cualquier otro caso. Hay que considerar que cada ecuación tiene los mismos regresores, esto es, los valores rezagados de $y_{t}$ y $u_{t}$. Bajo esta especificación se considera que los niveles pasados del desempleo y el producto pueden afectar 
el nivel actual de la producción. Los coeficientes $\pi_{1 j}$ y $\pi_{2 j}, j=1,2$ capturan el efecto de cambios en la producción y $\gamma_{1 j}$ y $\gamma_{2 j}$ y $j=1,2$ miden los efectos de cambios en el desempleo.

Las relaciones entre las variables en un modelo VAR son difíciles de establecer directamente desde las matrices de parámetros (Lütkepohl, 2010). La interdependencia y estructura dinámica de las variables en un modelo VAR implica que los cambios en una variable repercuten en todas las variables del sistema, y que se produzcan respuestas contemporáneas, así como, en los periodos subsiguientes. Por esto, se han propuesto las funciones de impulso respuesta (FIR) como herramientas para analizar las relaciones entre las variables en modelos VAR (Lütkepohl, 2010). La FIR mide la respuesta de cada una de las variables ante choques en los términos de error de las variables del modelo, durante varios periodos en el futuro. Para calcular las FIR se parte del modelo VAR(p) de la ecuación 2 que se puede expresar como $\Pi(L) X_{t}=\varepsilon_{t}$ donde $L$ representa un operador de rezago y $\Pi(L)$ es una matriz polinomial en el operador de rezago definida como $\Pi(L)=I_{N}-\Pi_{1} L-\cdots-\Pi_{p} L^{p}$. A partir de la representación de media móvil del modelo $\operatorname{VAR}(\mathrm{p})$, se determina la FIR:

$$
X_{t}=\Pi(L)^{-1} \varepsilon_{t}=\varepsilon_{t}+\sum_{i=1}^{\infty} \varphi_{i} \varepsilon_{t-i}
$$

Donde la matriz $\varphi_{i}$ mide la respuesta de $X_{t}$ a un impulso en $\varepsilon_{t}$ a medida que transcurre el tiempo.

\section{Cointegración}

Si las variables en el modelo 3 son I(1) entonces los resultados estadísticos usuales pueden no ser válidos, al menos de que exista una relación de equilibrio de largo plazo entre las series (Maddala \& Kim, 1998). Las variables mantienen una relación de equilibrio cuando las series comparten la misma tendencia común. A estas relaciones se interpretan como de equilibrio de largo plazo porque las fuerzas económicas que actúan en respuesta a desviaciones toman tiempo para restaurar el equilibrio. Para establecer una relación de largo plazo entre el nivel de producción y desempleo se utiliza la técnica de cointegración de Johansen \& Juselius (1990). Esta técnica de cointegración toma como punto de partida la representación de modelo de vector de corrección de error (VECM) del modelo VAR.

$$
\Delta X_{t}=\alpha+\Pi X_{t-1}+\Gamma_{1} \Delta X_{t-1}+\cdots+\Gamma_{p-1} \Delta X_{t-p+1}+\varepsilon_{t}
$$

Donde $\Pi$ representa el impacto de largo plazo cuyo rango determina el número de vectores de cointegración ( $\mathrm{r}$ ), definida como $\Pi=\Pi_{1}+\cdots+\Pi_{p}-\mathrm{I}_{n}$. La matriz $\Gamma_{k}$ es el impacto de corto plazo y está definida como $\Gamma_{k}=-\sum_{j=k+1}^{p} \Pi_{j}, k=1, \ldots, p-1$. Se puede expresar la matriz $\boldsymbol{\Pi}$ en términos de una matriz de vectores de cointegración $\boldsymbol{\beta}$ (cuyas filas forman los $\mathrm{r}$ vectores de cointegración) y una matriz de parámetros de ajuste $\boldsymbol{\alpha}$ (cuyos elementos distribuyen el impacto de los vectores de cointegración a la evolución de $\Delta X_{t}$ ), tal que $\boldsymbol{\Pi}=\boldsymbol{\alpha} \boldsymbol{\beta}^{\prime}$. Johansen \& Juselius (1990) proponen dos diferentes pruebas conocidas como la prueba de la traza y la 
prueba de Maximum-eigenvalue para determinar el número de vectores de cointegración utilizando estimaciones de máxima verosimilitud.

A continuación, se presenta la estimación de los parámetros según la metodología señalada y se lleva a cabo el análisis de la función de impulso respuesta y las pruebas de causalidad de Granger.

La selección del número óptimo de rezagos en la estimación del modelo VAR se llevó a cabo a partir de los criterios de información Akaike, Schwarz y Hannan-Quinn. Los tres criterios concuerdan en dos rezagos como la estructura óptima (Anexo 2).

Los resultados de la estimación del modelo VAR se muestran en la tabla 4 y son presentados tal como son descritos por el modelo 3. La segunda y tercera columna de la tabla 4 presentan los resultados para el modelo del producto y desempleo, respectivamente. Para interpretar los resultados del modelo VAR, el gráfico 3 presenta la FIR estimada a partir de los resultados de la tabla 4. La figura 3a presenta la dinámica de la producción ante un ajuste exógeno de un incremento de la tasa de desempleo, conjuntamente con sus bandas de confianza al 95\%. Los resultados indican que el desempleo tiene un efecto negativo estadísticamente significativo sobre la producción, lo que señala que, ante un ajuste exógeno de la tasa de desempleo, el producto disminuye en alrededor de $0.51 \%$ luego de cinco trimestres. El producto responde a un choque positivo del desempleo siguiendo un patrón de $\mathrm{U}$ invertida. Luego de siete trimestres, el producto es $0.39 \%$ más bajo de lo que habría sido sin el efecto del desempleo. La respuesta del producto llega a un punto máximo en el quinto trimestre para posteriormente retornar a su nivel inicial. La función impulso respuesta también muestra que, frente a un choque en la tasa de desempleo, la producción responde de forma positiva a partir del trimestre doce, no obstante, este efecto no es estadísticamente significativo. La figura $3 \mathrm{~b}$ muestra la respuesta del desempleo ante choques positivos en el producto. De igual forma, se determina que el desempleo responde de forma contraria ante choques positivos en la producción. En este caso, se observa que el desempleo se ajusta más rápido que la producción frente a los choques que experimenta el sistema, puesto que el desempleo alcanza su punto máximo en el tercer trimestre, a partir de entonces el efecto no es estadísticamente significativo.

Tabla 4.

Estimación de modelo VAR

\begin{tabular}{lll}
\hline & Producción & Desempleo \\
\hline Producción(-1) & $0.980 * * *$ & $-23.137^{* *}$ \\
\hline Producción(-2) & $(0.155)$ & $(11.660)$ \\
\hline Desempleo(-1) & $-0.400^{* * *}$ & $28.589^{* * *}$ \\
\hline & $(0.133)$ & $(10.315)$ \\
\hline Desempleo(-2) & $-0.002^{*}$ & $0.366^{* *}$ \\
\hline Constante & $(0.001)$ & $(0.157)$ \\
\hline & $-0.005^{* *}$ & $0.371^{* *}$ \\
\hline R cuadrado & $(0.002)$ & $(0.156)$ \\
\hline Chi cuadrado (P>chi2) & -0.00003 & -0.017 \\
\hline
\end{tabular}

Esta obra se comparte bajo la licencia Creative Common Atribución-No Comercial 4.0 International (CC BY-NC 4.0) Revista de la Universidad Internacional del Ecuador. URL: https://www.uide.edu.ec/ 


\begin{tabular}{lll}
\hline & Producción & Desempleo \\
\hline $\mathbf{N}$ & 50 & 50 \\
\hline Log de verosimilitud & 133.0124 & 134.047 \\
\hline
\end{tabular}

$* \mathrm{p}<0.10, * * \mathrm{p}<0.05, * * * \mathrm{p}<0.01$. Entre paréntesis se presentan los errores estándar.

Fuente: Elaboración propia.

\section{Figura 3 a}

Respuesta del producto a un shock en el desempleo.

0,006

0,004

0,002

0,000

$-0,002$

$-0,004$

$-0,006$

$-0,008$

$-0,010$

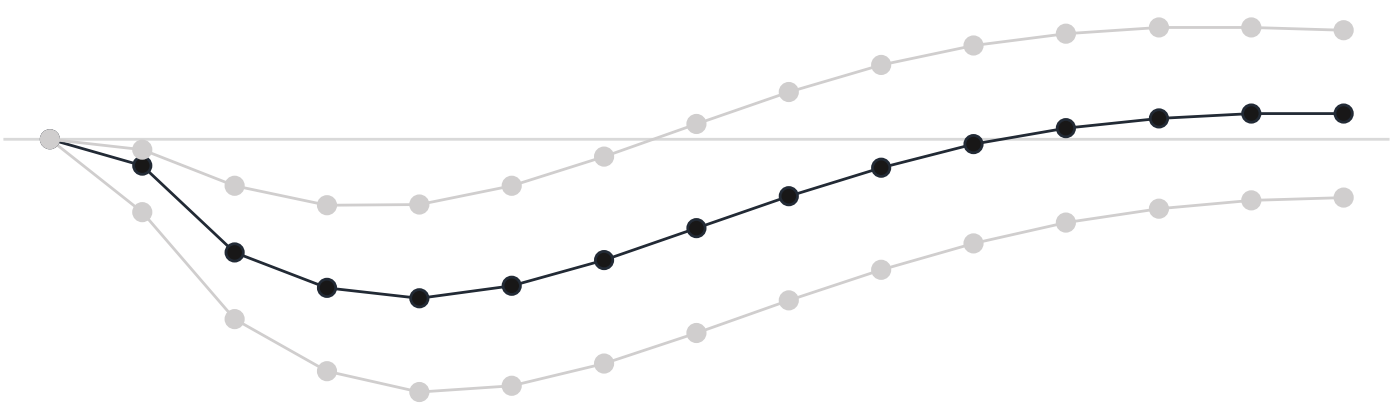

$\begin{array}{lllllllllllllll}1 & 2 & 3 & 4 & 5 & 6 & 7 & 8 & 9 & 10 & 11 & 12 & 13 & 14 & 15\end{array}$

- Límite inferior $\longrightarrow$ - Respuesta $\longrightarrow$ Límite superior

Se describe la respuesta de la producción a un shock positivo de una desviación estándar (Cholesky dof adjusted) en la tasa de desempleo. Los intervalos de confianza se calculan con un nivel de confianza del 95\%, y los errores estándar de las respuestas se estiman a través del método de Monte Carlo con 1000 repeticiones.

Fuente: Elaboración propia.

\section{Figura 3b}

Respuesta del desempleo a un shock en el producto.

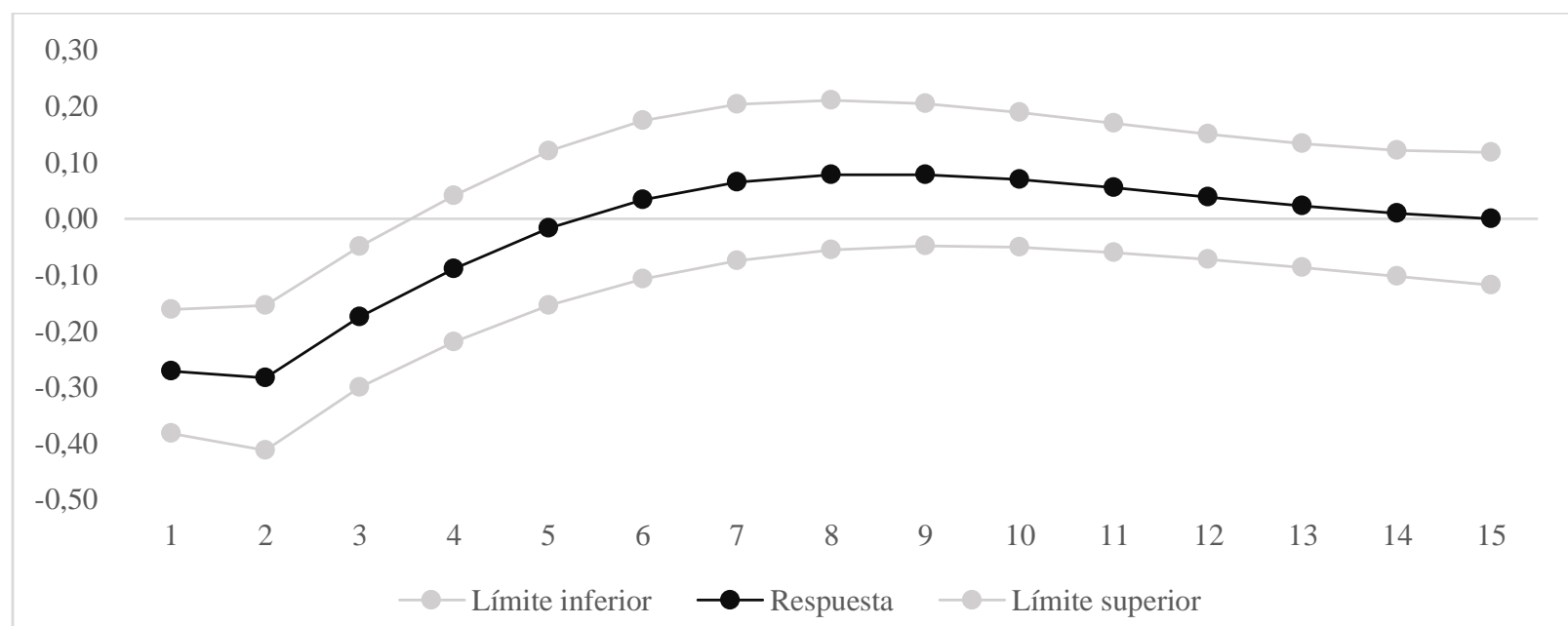

Se describe la respuesta del desempleo a un shock positivo de una unidad desviación estándar (Cholesky dof adjusted) en el producto. Los intervalos de confianza se calculan con un nivel de confianza del 95\%, y los errores estándar de las respuestas se estiman a través del método de Monte Carlo con 1000 repeticiones.

Fuente: Elaboración propia. 
Conocer la dirección de causalidad entre las variables es fundamental puesto que sugiere la mejor forma de alcanzar los objetivos de política de mayor crecimiento y bajo desempleo. Para esto se aplica el enfoque de causalidad de Granger que se basa en probar la significancia de los coeficientes de los rezagos de las variables que fueron consideradas como regresores en el modelo VAR. El análisis de causalidad presentado en la tabla 5 indica que existe una relación causal bidireccional entre el producto y el desempleo. En este sentido, el efecto de retroalimentación entre las dos variables significa que el incremento de la tasa de desempleo afecta el producto, pero los cambios de la producción también repercuten sobre el nivel de empleo. Esto se explica porque la causalidad de Granger también va desde el crecimiento económico al desempleo.

Tabla 5.

Análisis de causalidad

\begin{tabular}{llc}
\hline \multirow{2}{*}{ Regresor } & \multicolumn{1}{l}{ Variable dependiente } \\
\cline { 2 - 3 } & Desempleo & Producto \\
\hline Producción & $8.835 *$ & $8.020 * *$ \\
\hline Desempleo & & \\
$* \mathrm{p}<0.10, * * \mathrm{p}<0.05, * * * \mathrm{p}<0.01$. Presenta el estadístico Chi cuadrado para la prueba de hipótesis de que el regresor \\
causa en el sentido de Granger a la variable dependiente. \\
$\begin{array}{l}\text { Fuente: Elaboración propia. }\end{array}$
\end{tabular}

A continuación, se lleva a cabo el análisis de cointegración para determinar la relación de equilibrio de largo plazo entre la producción y el desempleo. El resumen de las pruebas de cointegración de Johansen \& Juselius (1990) se presenta en la tabla 6. Las pruebas se llevan a cabo considerando un término constante, pero no una tendencia. Las pruebas indican la existencia de una relación de cointegración entre las dos variables; específicamente, los resultados determinan un vector de cointegración.

Tabla 6.

Resumen de las pruebas de cointegración de Johansen-Juselius

\begin{tabular}{llll}
\hline Tipo de prueba & Estadístico & Valor crítico & $\begin{array}{l}\text { Núm. de relaciones de } \\
\text { cointegración }\end{array}$ \\
\hline Traza & 25.79 & 15.41 & 1 \\
\hline Maximum-eigenvalue & 16.14 & 14.07 & 1 \\
\hline
\end{tabular}

Número de relaciones de cointegración determinadas por el modelo al nivel de significancia de 5\%. El estadístico hace referencia a la prueba con la hipótesis nula de que hay cero ecuaciones de cointegración. Las relaciones de cointegración se determinan considerando un término constante. Orden de las variables: PIB, desempleo.

Fuente: Elaboración propia.

La tabla 7 presenta el modelo VECM estimado. Como se esperaba, el término de corrección de error (ECT) es negativo, lo que indica que el modelo converge al equilibrio después de una perturbación en el sistema. Con un nivel de confianza del 95\%, el valor del ECT es estadísticamente diferente de cero. Específicamente, el ECT para DProducción indica que aproximadamente el $39.77 \%$ de la desviación con respecto al equilibrio es corregido dentro de un trimestre. En el caso de DDesempleo el ECT presenta un signo negativo, pero no es 
estadísticamente significativo. El valor del ECT indica que alrededor del $60.17 \%$ del desequilibrio se corrige en un trimestre.

\section{Tabla 7.}

Estimación de modelo de Vector de Corrección de Error

\begin{tabular}{lll}
\hline & DProducción & Ddesempleo \\
\hline DProducción(-1) & $0.354 * *$ & -186.408 \\
\hline & $(0.135)$ & -113.017 \\
\hline DDesempleo(-1) & $0.005^{* *}$ & $-0.450 * *$ \\
\hline ECT & $(0.002)$ & $(0.176)$ \\
\hline Constante & $-0.398^{* * *}$ & -0.602 \\
\hline & $(0.101)$ & -85.187 \\
\hline
\end{tabular}

$* \mathrm{p}<0.10, * * \mathrm{p}<0.05, * * * \mathrm{p}<0.01$. Entre paréntesis se presentan los errores estándar. Fuente: Elaboración propia.

Siguiendo la metodología de Johansen \& Juselius (1990), la relación de cointegración de largo plazo normalizada es la siguiente:

$$
\begin{gathered}
y=-0.004-0.0206 u \\
\text { error estándar } \quad(0.004) \\
z
\end{gathered}
$$

Los signos observados son como los anticipados, y se determina que el coeficiente de Okun es estadísticamente significativo a un nivel de significancia del 1\%. Así, los resultados obtenidos por este enfoque son consistentes con la ley de Okun. Dado que la producción está expresada en logaritmo, se puede interpretar el coeficiente estimado $\left(\beta_{1}\right)$ como incrementos porcentuales. En este sentido, los resultados de la ecuación de cointegración indican que el incremento de 1 p.p. de la tasa de desempleo, genera que la producción disminuya en promedio en $2.06 \%$. Este resultado es mayor al obtenido por el modelo estático.

\section{Enfoque de cointegración ARDL}

A continuación se lleva a cabo el análisis de la relación de largo plazo entre el producto y el desempleo a partir del enfoque ARDL propuesto por Pesaran \& Shin (1995) y Pesaran, Shin \& Smith (2001). Como se mencionó anteriormente, entre las principales razones para el uso de esta metodología se encuentran: a) el procedimiento es aplicable irrespectivamente de si las variables son I(0), I(1) o mutuamente cointegradas y, por lo tanto, no es necesario determinar de forma previa el grado de integración de las variables en el modelo; b) permite que las variables tengan diferentes rezagos óptimos; y, c) la literatura empírica prefiere este enfoque cuando la muestra es relativamente pequeña debido a que se basa en una sola ecuación de forma reducida para estimar simultáneamente los efectos de corto y largo plazo. La forma de corrección de error del modelo ARDL correspondiente a la ecuación 1 se resume en la siguiente ecuación: 


$$
\begin{gathered}
\Delta y_{t}=\alpha+\phi y_{t-1}+\beta u_{t-1}+\sum_{j=1}^{p-1} \gamma_{j} \Delta y_{t-j} \\
+\sum_{j=0}^{q-1} \delta_{j} \Delta u_{t-j}+\varepsilon_{t} \\
\Delta y_{t}=\alpha+\phi\left[y_{t-1}-\theta u_{t-1}\right]+\sum_{j=1}^{p-1} \gamma_{j} \Delta y_{t-j} \\
+\sum_{j=0}^{q-1} \delta_{j} \Delta u_{t-j}+\varepsilon_{t}
\end{gathered}
$$

Donde el término $\theta=-\frac{\beta}{\phi}$ define la relación de largo plazo entre la producción y el desempleo, y $\delta_{j}$ la relación de corto plazo. El término $\phi$ mide la velocidad de ajuste de la producción hacia su equilibrio de largo plazo siguiendo un cambio dado en el desempleo, y $\phi<$ 0 asegura que dicha relación de largo plazo exista. Determinar un coeficiente $\phi$ negativo y significativo puede ser evidencia de una relación de cointegración entre la producción y el desempleo. Con base en el modelo de corrección de error expresado en la ecuación anterior, se prueba la hipótesis nula de no cointegración $(\phi=0$ y $\beta=0)$ utilizando una prueba $\mathrm{F}$. La prueba utilizada para este procedimiento no tiene una distribución estándar. Pesaran, Shin \& Smith (2001) calculan los valores críticos asintóticos para la prueba de cointegración para diferentes niveles de significancia, para cinco casos de interés dependiendo de la especificación de los términos determinísticos. Un conjunto de valores críticos asume que todas las variables son I(0) y el otro asume que todas son I(1). Es decir, estos valores críticos incluyen una banda superior e inferior que proporcionan un rango que cubre todas las clasificaciones posibles de las variables en I(0) y I(1). Si el estadístico F calculado se encuentra por arriba del nivel superior de la banda, se rechaza la hipótesis nula, y se concluye que las variables cointegran. Si el estadístico F calculado se ubica por debajo del nivel inferior de la banda, la hipótesis nula no puede ser rechazada. Si el estadístico se encuentra dentro de la banda, entonces el resultado es inconclusivo.

La tabla 8 presenta la representación de corrección de error del modelo ARDL. Se toma como punto de partida 4 rezagos en cada variable explicativa, y luego se procedió a determinar la longitud óptima del rezago con base en el criterio de información de Akaike. El resultado fue un $\operatorname{ARDL}(2,2)$.

Los estadísticos $\mathrm{F}$ y t de la tabla 8 hacen referencia a los estadísticos para probar la existencia de una relación de largo plazo entre la producción y desempleo; la prueba incluye intercepto, pero no tendencia. El estadístico F y t de la prueba de cointegración de 8.672 y -4.051 se encuentran por arriba de la banda superior para al 5\%, la hipótesis nula es rechazada y se concluye que las variables se encuentran cointegradas. Como se esperaba, el término de corrección de error es negativo y estadísticamente significativo, e indica que alrededor del $39.37 \%$ del desequilibrio en el producto con respecto a su nivel de largo plazo se corrige en un 
trimestre. En este caso se determina que el incremento de 1 p.p. en la tasa de desempleo genera que la producción disminuya en promedio en $2.09 \%$.

Tabla 8.

Representación de corrección de error del modelo ARDL

\begin{tabular}{ll}
\hline Variable & \\
\hline Desempleo(-1) & $-0.0209^{* * * *}$ \\
& $(0.004)$ \\
DProducción(-1) & $0.261^{*}$ \\
& $(0.141)$ \\
DDesempleo & 0.003 \\
& $(0.003)$ \\
DDesempleo(-1) & 0.003 \\
& $(0.002)$ \\
Constante & -0.0001 \\
& $(0.001)$ \\
ECT & $-0.394^{* * *}$ \\
& $(0.097)$ \\
$\mathbf{F}^{\mathbf{c}}$ & 8.672 \\
$\mathbf{t}^{\mathbf{c}}$ & -4.051 \\
$\mathbf{R}$ cuadrado & 0.363 \\
Log de verosimilitud & 177.13 \\
$\mathbf{N}$ & 50 \\
\hline
\end{tabular}

$* \mathrm{p}<0.10, * * \mathrm{p}<0.05, * * * \mathrm{p}<0.01$. Entre paréntesis se presentan los errores estándar. a denota que el estadístico se ubica por debajo límite inferior del 95\%, b denota que este cae dentro de los límites del 95\%, y c denota que este se ubica fuera del 95\% de límite superior. Los valores críticos fueron tomados de Kripfganz \& Schneider (2018); los límites superior e inferior fueron de 5.093 y 6.008 para la prueba $F$, y de -2.890 y -3.267 para la prueba t.

Fuente: Elaboración propia.

Con el propósito de establecer la sensibilidad de los resultados a la elección del filtro, el anexo 3 también presenta las estimaciones obtenidas con el filtro band-pass de Baxter-King. Los resultados confirman la existencia de una relación negativa de largo plazo entre la producción y el desempleo. En este caso, se encuentra que el impacto del desempleo sobre el producto es menor, lo que puede responder al menor periodo de tiempo al que hace referencia los resultados (2010.I-2016.IV), puesto que la aplicación del filtro de Baxter-King implica la pérdida de doce observaciones al inicio y al final de la serie.

\section{Conclusiones}

El estudio analiza la ley de Okun para el caso de Ecuador. Con este objetivo, se aplica un análisis de cointegración con datos trimestrales para el periodo 2007.I y 2019. IV. El análisis se lleva a cabo a partir de dos enfoques de cointegración, la prueba de cointegración de Johansen \& Juselius (1990) y el enfoque basado en un modelo ARDL. El análisis empírico de la ley de Okun empieza por un análisis estático, el cual indica una relación negativa estadísticamente significativa entre el producto y el desempleo.

Con respecto al análisis de cointegración, se observa que los resultados son robustos entre las metodologías utilizadas. El análisis confirma la existencia de una relación de cointegración de largo plazo entre la producción y desempleo, y que dicha relación es negativa, lo cual es 
consistente con la ley de Okun. Los resultados de la ecuación de cointegración obtenidos a partir del enfoque de Johansen \& Juselius (1990) señalan que un punto porcentual adicional en la tasa de desempleo disminuye la producción, en promedio, en 2.06\%. En el caso del enfoque ARDL, los resultados señalan que el efecto del desempleo sobre el producto es de $2.09 \%$. En este sentido, se encuentra evidencia a favor de la ley de Okun para el caso de Ecuador. Cabe señalar que las estimaciones del coeficiente de Okun para Ecuador obtenidas en el presente estudio son más bajas a las observadas en países desarrollados. Esto puede responder a diferentes factores como la rigidez laboral y a características del mercado laboral ecuatoriano como la existencia de un elevado subempleo, empleo inadecuado e informalidad laboral. De acuerdo con el INEC (2020) en diciembre del 2019, el $17.8 \%$ de empleados están subempleados, tan solo el $38.8 \%$ tienen un empleo adecuado y el 46,7\% se encuentran en el sector informal de la economía. Estos factores pueden minimizar el impacto de las fluctuaciones del producto sobre el nivel de desocupación.

El análisis también muestra la existencia de una relación de causalidad bidireccional entre el producto y el desempleo. Esto implica que el aumento del desempleo influye en el producto, pero al mismo tiempo los cambios en la producción repercuten sobre el nivel de empleo. Se observa que el producto responde a un choque positivo del desempleo siguiendo un patrón de U invertida. El impacto del desempleo llega a su nivel máximo en el quinto trimestre para posteriormente retornar a su nivel original. También, se determina que el desempleo responde de forma inversa ante un incremento en la producción; alcanzando su efecto máximo en el tercer trimestre.

En este sentido, se pueden sugerir varias implicaciones de política de los resultados obtenidos. Primero, los hacedores de política deben considerar la generación de un crecimiento sostenido como una estrategia clave para combatir el desempleo en el país. Segundo, la necesidad de considerar el elevado costo en términos de producto de la desocupación. Tercero, los hacedores de políticas deben considerar que los efectos en la producción y el empleo se transmiten a lo largo de un determinado número de períodos. Esta consideración resulta clave al momento de analizar el impacto de políticas orientadas a promover la producción y empleo, puesto que se pueden observar tanto efectos contemporáneos, como en periodos futuros. Cuarto, los resultados también ponen de manifiesto la necesidad de considerar las distintas velocidades de ajuste de la producción y el desempleo ante choques en la economía; en el caso de la producción el efecto de un choque alcanza su nivel máximo en el quinto trimestre, mientras que el efecto en el desempleo alcanza su punto máximo en el tercer trimestre.

Los resultados tienen, por supuesto, algunas limitaciones. La principal limitación del análisis es el tamaño del periodo de estudio, lo que responde a la disponibilidad de información comparable. Por esto, se decidió contrastar los resultados con la metodología de cointegración basada en un modelo ARDL que es preferida cuando el número de observaciones es limitado, lo que representa un valor añadido del estudio. Adicionalmente, se considera que un aspecto que permanece sin abordar en este análisis es la importancia de otros factores que pueden mediar en la relación entre la actividad económica y el desempleo. Por ejemplo, se ha discutido que, en países en desarrollo, el grado de flexibilidad del mercado laboral puede incidir en la respuesta del desempleo ante fluctuaciones de la producción. El análisis de estos factores, no obstante, requiere un periodo de tiempo más prolongado debido a que los métodos de series de tiempo 
multivariadas generan un número de parámetros a estimar que aumenta rápidamente con las variables y rezagos considerados, lo que en el presente caso dificulta obtener un análisis estadístico significativo. Ciertamente, la importancia de estos factores para el caso ecuatoriano debe ser considerada en investigaciones futuras.

\section{Bibliografía}

Abu, N. (2017). Does okun's law exist in Nigeria? Evidence from the ardl bounds testing approach. Contemporary Economics, 11(2), 131-144. https://doi.org/10.5709/ce.1897-9254.232

Adanu, K. (2005). A cross-province comparison of Okun's coefficient for Canada. Applied Economics, 37(5), 561-570. https://doi.org/10.1080/0003684042000201848

Apergis, N., \& Rezitis, A. (2003). An examination of Okun's law: Evidence from regional areas in Greece. Applied Economics, 35(10), 1147-1151. https://doi.org/10.1080/0003684032000066787

Attfield, C. L. F., \& Silverstone, B. (1997). Okun's Coefficient: A Comment. Review of Economics and Statistics, 79(2), 326-329. https://doi.org/10.1162/003465397556692

Ball, L., Furceri, D., Leigh, D., \& Loungani, P. (2019). Does One Law Fit All? Cross-Country Evidence on Okun's Law. Open Economies Review, 30(5), 841-874. https://doi.org/10.1007/s11079-019-09549-3

Barreto, H., \& Howland, F. (1993). There are two Okun's law relationships between output and unemployment. Manuscript (faculty works, Wabash college), (317), 1-27.

Baxter, M., \& King, R. G. (1995). Approximate Band-Pass Filters for Economic Time Series. NBER Working Paper Series, 5022, 1-53.

BCE. (2019). Publicación No. 24 Retropolación 1965-2006 y Cuentas Nacionales Anuales 2007. Recuperado 10 de octubre de 2019, de https://contenido.bce.fin.ec/documentos/PublicacionesNotas/Catalogo/CuentasNacional es/Bol_retro24.xlsx

BCE. (2020a). Boletín de Cuentas Nacionales Trimestrales. Recuperado 1 de abril de 2020, de https://contenido.bce.fin.ec/home1/estadisticas/cntrimestral/CNTrimestral.jsp

BCE. (2020b). Ciclo económico del Ecuador. Resultados al cuarto trimestre del 2020. Recuperado 1 de marzo de 2020, de 2020 website: https://contenido.bce.fin.ec/documentos/Estadisticas/SectorReal/Previsiones/IDEAC/Ci cloEconIVT2019.pdf

Briceño, M., Dávila, G., \& Rojas, M. . (2017). Estimación de la Ley de Okun: evidencia empírica para Ecuador, Amé rica Latina y el Mundo. Revista Económica, 1, 35-45. Recuperado de https://revistas.unl.edu.ec/index.php/economica/article/view/209

Chavarín, R. (2001). El costo del desempleo medido en producto: Una revisión empírica de la ley de Okun para México. Trimestre Economico, 68(2), 209-231. https://doi.org/10.2307/20857058

Elliott, G., Rothenberg, T., \& Stock, J. (1992). Efficient tests for an autoregressive unit root. Recuperado de https://www.nber.org/papers/t0130

Flórez, L. A., Pulido-Mahecha, K. L., Ramos, M. A., \& Ramos-Veloza, M. A. (2018). Okun`s law in Colombia: a non-linear cointegration OKUN'S LAW IN COLOMBIA: A NONLINEAR COINTEGRATION APPROACH. En repositorio.banrep.gov.co. Recuperado de http://repositorio.banrep.gov.co/handle/20.500.12134/7010

Hodrick, R. J., \& Prescott, E. C. (1997). Postwar U.S. Business Cycles: An Empirical 
Investigation. Journal of Money, Credit and Banking, 29(1), 1. https://doi.org/10.2307/2953682

INEC. (2020). Encuesta Nacional de Empleo, Desempleo y Subempleo. Indicadores laborales diciembre del 2019. Recuperado 1 de abril de 2020, de https://www.ecuadorencifras.gob.ec/documentos/webinec/EMPLEO/2019/Diciembre/2 01912_Mercado_Laboral.pdf

Islas, A. C., \& Cortez, W. W. (2013). Relaciones dinámicas del producto y el empleo en México: Una evaluación de sus componentes permanentes y transitorios. Cepal Review, (111), 167-182. https://doi.org/10.18356/090d56b2-es

Islas C., A., \& Cortez, W. W. (2019). ¿Puede el sector informal afectar a la relación entre desempleo y producción? Un análisis del caso de México. Revista de la CEPAL, 2018(126), 151-169. https://doi.org/10.18356/187829c9-es

Johansen, S., \& Juselius, K. (1990). MAXIMUM LIKELIHOOD ESTIMATION AND INFERENCE ON COINTEGRATION - WITH APPLICATIONS TO THE DEMAND FOR MONEY. Oxford Bulletin of Economics and Statistics, 52(2), 169-210. https://doi.org/10.1111/j.1468-0084.1990.mp52002003.x

Kripfganz, S., \& Schneider, D. (2018). Introduction ARDL model EC representation Bounds testing Postestimation Further topics Summary ardl: Estimating autoregressive distributed lag and equilibrium correction models. stata-uk.com, 1-44. Recuperado de http://www.kripfganz.de/stata/

Larrea, C. (2004). Crisis, dolarización y pobreza en el Ecuador. Retos para la integración social de los pobres en América Latina. CLACSO.

Loria Diaz De Guzman, E. G., Ramírez Guerra, E. A., \& Salas, E. (2015). La Ley de Okun y la flexibilidad laboral en México: Un análisis de cointegración, 1997Q3-2014Q1. Contaduria y Administracion, 60(3), 631-650. https://doi.org/10.1016/j.cya.2015.05.012

Lütkepohl, H. (2010). Impulse response function. En Macroeconometrics and Time Series Analysis (pp. 145-150). https://doi.org/10.1057/9780230280830_16

Maddala, G., \& Kim, I. (1998). Unit roots, cointegration, and structural change. Cambridge University Press.

Mendieta Muñoz, R. (2015). Remesas y disparidades económicas territoriales. Universidad de Cuenca.

Nourzad, F., \& Almaghrbi, Y. (1996). Okun's law and the fulfillment of wage and price expectations. Journal of Post Keynesian Economics, 18(2), 293-308. https://doi.org/10.1080/01603477.1995.11490073

OIT. (2014). Evolución del empleo informal en Ecuador. Center for economic and policy research. Okun, A. (1963). Potential GNP: its measurement and significance. Cowless Foundation.

Pesaran, M., \& Shin, Y. (1995). Long-run structural modelling (No. 9419). Faculty of Economics, University of Cambridge.

Pesaran, M., Shin, Y., \& Smith, R. (2001). Bounds testing approaches to the analysis of level relationships. Journal of Applied Econometrics, 16(3), 289-326. https://doi.org/10.1002/jae.616

Prachowny, M. F. J. (1993). Okun's Law: Theoretical Foundations and Revised Estimates. The Review of Economics and Statistics, 75(2), 331. https://doi.org/10.2307/2109440

Rodríguez, A., Hurtado, C., \& Vega, N. (2017). Aplicación de la Ley de Okun para Ecuador: un análisis de la producción y su incidencia en la tasa de desempleo. UIDE, 247-263.

Schwert, G. W. (2002). Tests for unit roots: A Monte Carlo investigation. Journal of Business and

Esta obra se comparte bajo la licencia Creative Common Atribución-No Comercial 4.0 International (CC BY-NC 4.0) Revista de la Universidad Internacional del Ecuador. URL: https://www.uide.edu.ec/ 
Economic Statistics, 20(1), 5-17. https://doi.org/10.1198/073500102753410354

Senplades. (2016). Evalución de los Costos de Reconstrucción Sismo en Ecuador abril 2016.

SIISE. (2019). Desigualdad y Pobreza. Consultas Temáticas. Recuperado 10 de octubre de 2019, de http://www.siise.gob.ec/siiseweb/siiseweb.html?sistema=1.

Wong, S. A. (2019). Minimum wage impacts on wages and hours worked of low-income workers in Ecuador. World Development, $116, \quad$ 77-99. https://doi.org/10.1016/j.worlddev.2018.12.004

Zagler, M. (2003). A VECTOR ERROR CORRECTION MODEL OF ECONOMIC GROWTH AND UNEMPLOYMENT IN MAJOR EUROPEAN COUNTRIES AND AN ANALYSIS OF OKUN'S LAW. En Applied Econometrics and International Development. AEEADE (Vol. 3). Recuperado de https://papers.ssrn.com/sol3/papers.cfm?abstract_id=1229602

Zivot, E., \& Andrews, D. W. K. (2002). Further evidence on the great crash, the oil-price shock, and the unit-root hypothesis. Journal of Business and Economic Statistics, 20(1), 25-44. https://doi.org/10.1198/073500102753410372 


\section{Anexos}

\section{Anexo 1.}

Resultados de las pruebas de raíces unitarias (incluye intercepto)

\begin{tabular}{lllllll}
\hline & ADF & \multicolumn{2}{l}{ PP } & \multicolumn{2}{l}{ ERS } \\
\hline Variable & Nivel & $\begin{array}{l}\text { Primera } \\
\text { diferencia }\end{array}$ & Nivel & $\begin{array}{l}\text { Primera } \\
\text { diferencia }\end{array}$ & Nivel & $\begin{array}{l}\text { Primera } \\
\text { diferencia }\end{array}$ \\
\hline PIB & $-1.057(1)$ & $-3.503(0)^{*}$ & $-0.795(3)$ & $-4.749(3)^{* * *}$ & $-0.813(1)$ & $-3.406(0)^{* *}$ \\
Tasa de desempleo & $-1.940(2)$ & $-4.362(0)^{* * *}$ & $-2.705(3)^{*}$ & $-9.459(3)^{* * *}$ & $-0.978(2)$ & $-1.928(0)^{*}$
\end{tabular}

$* \mathrm{p}<0.10, * * \mathrm{p}<0.05, * * * \mathrm{p}<0.0$. Entre paréntesis se presenta la estructura de rezago óptima para la prueba ADF y DFGLS (determinado por el criterio de información de Akaike), para PP se presenta el número de rezagos de NeweyWest usados para calcular los errores estándar (determinada por la fórmula de Schwert, 1989). Las pruebas para el PIB incluyen tendencia.

Fuente: Elaboración propia.

Anexo 2.

Criterios de selección de la estructura óptima de rezagos para el modelo VAR

\begin{tabular}{lllll}
\hline Rezago & $\begin{array}{l}\text { Logaritmo de } \\
\text { Verosimilitud }\end{array}$ & AIC & SC & HQ \\
\hline $\mathbf{0}$ & 81.07 & -3.29 & -3.34 & -3.29 \\
$\mathbf{1}$ & 123.47 & -5.00 & -4.91 & -4.76 \\
$\mathbf{2}$ & 131.09 & -5.15 & -5.00 & -4.76 \\
$\mathbf{3}$ & 133.00 & -5.06 & -4.86 & -4.51 \\
$\mathbf{4}$ & 133.71 & -4.92 & -4.66 & -4.22 \\
\hline
\end{tabular}

Fuente: Elaboración propia.

Anexo 3.

Resultados obtenidos con el filtro Baxter-King

Anexo 3a. Resumen de las pruebas de cointegración de Johansen-Juselius

\begin{tabular}{llll}
\hline Tipo de prueba & Estadístico & Valor crítico & Núm. de relaciones de cointegración \\
\hline Traza & 48.022 & 15.41 & 1 \\
Maximum-eigenvalue & 31.8667 & 14.07 & 1 \\
\hline
\end{tabular}

Número de relaciones de cointegración determinadas por el modelo al nivel de significancia de 5\%. El estadístico hace referencia a la prueba con la hipótesis nula de que hay cero ecuaciones de cointegración. Las relaciones de cointegración se determinan considerando un término constante. Orden de las variables: PIB, desempleo.

Fuente: Elaboración propia.

\begin{tabular}{lll} 
Anexo 3b. Estimación de modelo de Vector de Corrección de Error & \\
\hline \multirow{2}{*}{ DProducción(-1) } & DProducción & DDesempleo \\
& $1.2613^{* * *}$ & $-24.8049^{* * * *}$ \\
DDesempleo(-1) & $(0.0875)$ & $(8.3117)$ \\
& $0.0116^{* * *}$ & $0.3324^{*}$ \\
ECT & $(0.0019)$ & $(0.1850)$ \\
& $-0.1262^{* * *}$ & 1.6933 \\
Constante & $(0.0255)$ & $(2.4260)$ \\
& $0.0013^{* * *}$ & 0.0001 \\
& $(0.0005)$ & $(0.0433)$ \\
\hline
\end{tabular}

Esta obra se comparte bajo la licencia Creative Common Atribución-No Comercial 4.0 International (CC BY-NC 4.0) Revista de la Universidad Internacional del Ecuador. URL: https://www.uide.edu.ec/ 
Ecuación de cointegración (variable dependiente PIB)

Desempleo

DProducción

DDesempleo

Constante

$-0.0115$

$-0.0067$

$* \mathrm{p}<0.10, * * \mathrm{p}<0.05, * * * \mathrm{p}<0.01$. Entre paréntesis se presentan los errores estándar.

Fuente: Elaboración propia.

\begin{tabular}{|c|c|}
\hline \multicolumn{2}{|c|}{$\begin{array}{l}\text { Anexo 3c. Representación de corrección de error del modelo ARDL } \\
\text { Variable }\end{array}$} \\
\hline Desempleo(-1) & $\begin{array}{l}-0.0132 * * * \\
(0.0042)\end{array}$ \\
\hline DProducción(-1) & $\begin{array}{l}1.0434 * * * \\
(0.0691)\end{array}$ \\
\hline DDesempleo & $\begin{array}{l}-0.0074 * * * \\
(0.0017)\end{array}$ \\
\hline DDesempleo(-1) & $\begin{array}{l}0.0148 * * * \\
(0.0016)\end{array}$ \\
\hline Constante & $\begin{array}{l}0.0005 * * \\
(0.0002)\end{array}$ \\
\hline ECT & $\begin{array}{l}-0.1160 * * * \\
(0.0192)\end{array}$ \\
\hline $\mathbf{F}^{\mathbf{c}}$ & 23.987 \\
\hline $\mathbf{t}^{\mathrm{c}}$ & -6.035 \\
\hline $\mathbf{R}$ cuadrado & 0.9754 \\
\hline Log de verosimilitud & 141.3666 \\
\hline $\mathbf{N}$ & 26 \\
\hline
\end{tabular}

$* \mathrm{p}<0.10, * * \mathrm{p}<0.05, * * * \mathrm{p}<0.01$. Entre paréntesis se presentan los errores estándar. a denota que el estadístico se ubica por debajo límite inferior del 95\%, b denota que este cae dentro de los límites del 95\%, y c denota que este se ubica fuera del 95\% límite superior. Los valores críticos fueron tomados de Kripfganz and Schneider (2018); los límites superior e inferior fueron de 6.017 y 5.099 para la prueba $F$, y de -3.269 y -2.891 para la prueba $t$.

Fuente: Elaboración propia. 